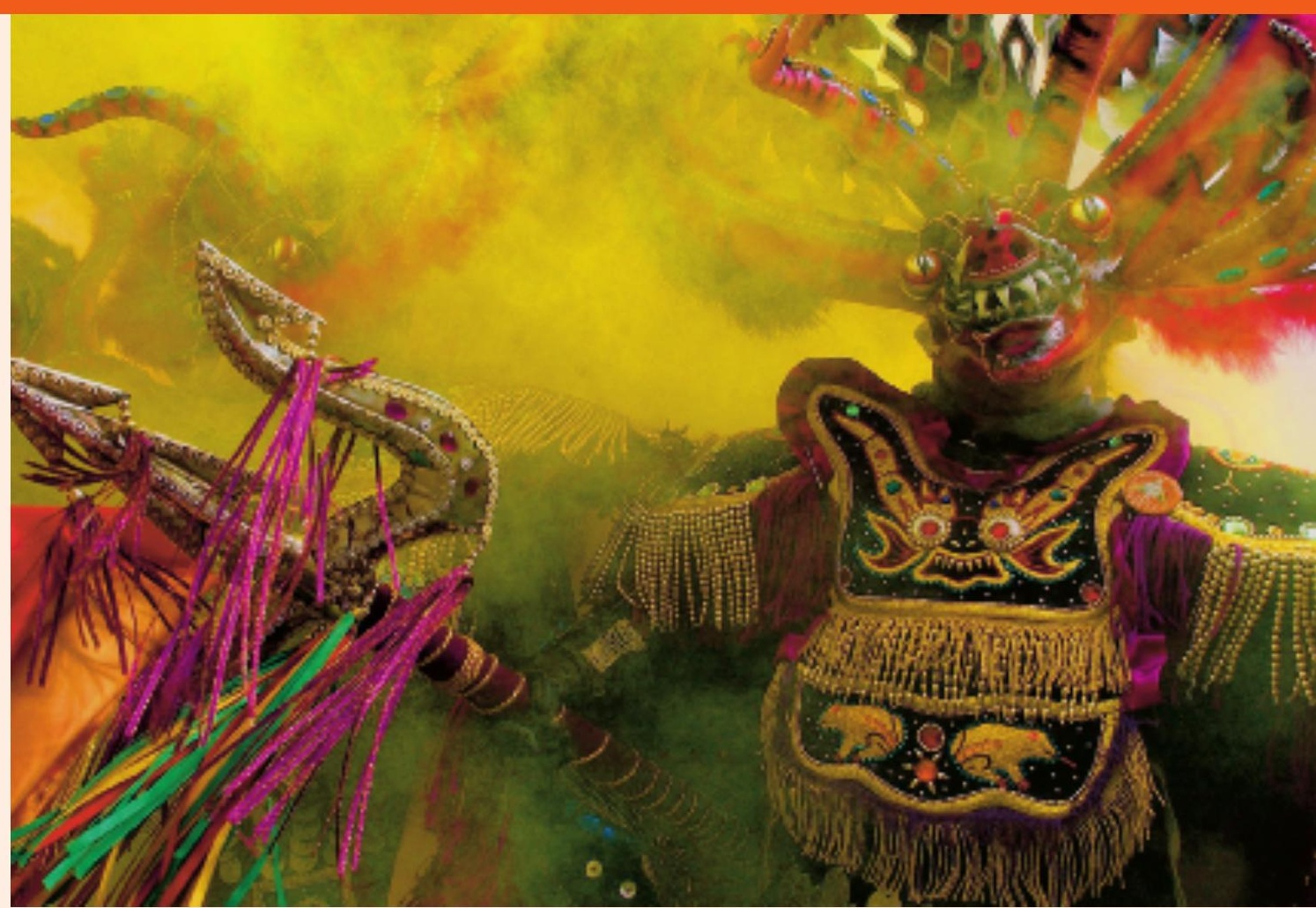

Höllischer Auftritt: Beim Karneval von Oruro, einem Unesco-Weltkulturerbe, stehen Luzifer und Konsorten im Mittelpunkt.

Bild: Dado Galdieri / Keystone.

\title{
Wo tausend Teufel tanzen
}

Während 51 Wochen im Jahr ist Oruro nicht viel mehr als ein verschlafenes Nest auf dem Altiplano, berühmt bestenfalls für seine unwirtliche Kälte und den permanenten Wassermangel. Alljährlich im Februar aber wird die unscheinbare Minenstadt für Bolivianer und andere Eingeweihte zum Nabel der Welt: Zur Karnevalszeit ist in Oruro buchstäblich die Hölle los.

Bruno Kesseli

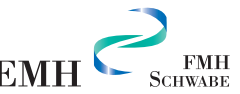

Feingewobene Schleier von Nieselregen schieben sich über den zentralen Altiplano, diese gigantische, auf rund 4000 Metern über Meer gelegene Hochebene zwischen der Ost- und der Westkette der Anden. Grau- und Brauntöne, soweit das Auge reicht, am wolkenverhangenen Himmel genauso wie in der zerklüfteten, erdig-felsigen Landschaft, deren Austrocknungsgrad durch den Wasserstaub keine wesentliche Korrektur erfahren wird. Ein Stimmung von melancholischer Poesie, zweifellos, doch eine Stimmung auch, die bei entsprechender Disposition subdepressive Gefühlslagen leicht in kompromisslose Schwermut umschlagen lässt.
Und schon sind wir mitten im Zentrum der bolivianischen Seele - derjenigen der Hochlandbewohner zumindest -, deren Eigenschaften sich in der Musik von Gruppen wie Los Kjarkas oder Savia Andina so wunderschön widerspiegeln. Da ist diese allgegenwärtige Wehmut, dieses fast existentialistische Gefühl des Ausgesetztseins, das, insbesondere wenn es in Kombination mit dem Schmerz nach gescheiterter Liebe auftritt, sich offenbar in vielen Fällen nur durch die Kombination von zwei Konstanten des andinen Lebensrhythmus ertragen lässt: Den Griff zur Flasche und Feste von aussergewöhnlicher Intensität und Farbenpracht. 


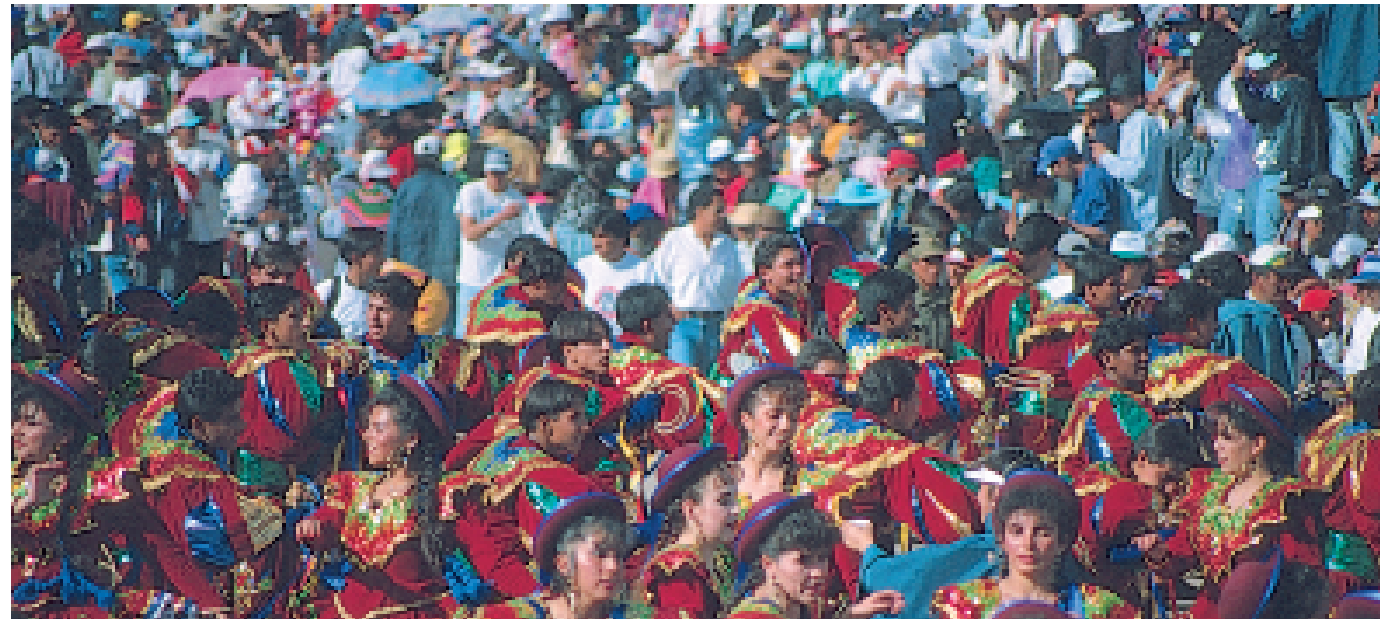

Kostümierte Tänzerinnen und Publikum vermischen sich zu einem bunten Volksfest.

\section{«Ich will tanzen, bevor ich sterbe»}

Beides werden wir in den kommenden Tagen hautnah erleben. Unser Weg führt nach Oruro, der vielbesungenen Minenmetropole, «dem grossen Zentrum», wie es in einem Lied von Jach'a Mallku, einer weiteren bolivianischen Formation von internationalem Ruf, heisst. «Ich will tanzen, bevor ich sterbe», wird da entschieden verkündet. Nicht nur in diesem Lied treten Tanz - als Inbegriff sinnlicher Vitalität - und Tod in einer Weise gemeinsam auf, die an das Lebensgefühl des Barock erinnert, wobei die Gegenwart des einen die Wahrnehmung und das Erleben des andern durch Kontrastwirkung intensiviert. Leben und Tod sind in diesem Teil des Altiplano als Gegensätze in einer Schärfe allgegenwärtig, die in den westlichen Kulturen durch konsequente Ausblendung des letzteren längst verlorengegangen ist.

Die Bewohner des zentralen Altiplano sind in bezug auf solche Erfahrungen allerdings schon landschaftsmässig im Vorteil. Bei der Gestaltung der Szenerie scheint der Schöpfer über weite Strecken mit den Elementen der «minimal art» experimentiert zu haben. Mineralisch-Totes, eine monotone Formen- und Farbenwelt dominiert, lässt aber die spärlich vorhandene Pflanzen- und Tierwelt um so deutlicher als etwas qualitativ «ganz anderes» hervortreten. Ein auf felsigem Untergrund gemächlich an kärglichen Gräsern rupfendes Vicuña springt den Betrachter in seiner Lebendigkeit geradezu an - das Gegenteil eines Videoclips, der durch permanente Reizüberflutung letztlich Abstumpfung bewirkt.

\section{Von Göttern und Teufeln}

Wo äussere Reize spärlich sind, kann die Innenwelt des Menschen sich ausbreiten, kann scheinbar Totes unversehens lebendig werden. Evoziert die Felsformation, die sich vor uns aufbaut, nicht das Bild eines gigantischen Frosches, erinnert jener Höhenzug seitlich dahinter nicht an eine kriechende Schlange? Die steinerne Ödnis beginnt zu erzählen von ihrer Vergangenheit, offenbart geduldigen Betrachtern und Zuhörern über Generationen ihre Geheimnisse. Die Welt von Oruro - so nennt sich auch das Departement, in dem die gleichnamige Stadt liegt - ist eine Welt der Mythen und Legenden, in denen uralte andine Kosmologie mit neuerem, katholischem Glaubensgut zu einer einzigartigen Mischung verschmolzen ist. Das Schattenreich der Minen und Höhlen tief in den Eingeweiden von Pachamama (Mutter Erde), aus denen Generationen von Ureinwohnern zunächst Silber, später Kupfer und Zinn zu schürfen gezwungen wurden, bildet ein im wörtlichen Sinne tiefgründiges Reservoir für über Jahrhunderte tradierte und kultivierte Geschichten.

Viele davon werden im Karneval von Oruro lebendig, dessen mythische Ursprünge weit in der prähispanischen Zeit liegen. In grauer Vorzeit sah sich das friedliebende Volk der Urus, das damals die unwirtliche Hochebene bewohnte, von Huari bedroht, einem Halbgott und Repräsentanten der finsteren Mächte. Doch die Urus hatten in der göttlichen Nusta eine hohe Beschützerin, die sich Huari unerschrocken entgegenstellte. Die Niederlage vor Augen, unternahm Huari einen letzten Versuch, das Blatt noch zu seinen Gunsten zu wenden. Er sandte eine Riesenschlange nach Süden, einen gigantischen Frosch nach Norden, eine gewaltige Echse nach Nordosten und eine Ameisenarmada nach Osten mit dem Auftrag, die Siedlungen der Urus vom Antlitz der Erde zu vertilgen. Nusta indessen war auf der Hut: Bevor die Todesboten ihre Mission erfüllen konnten, wurden sie durch 
die Göttliche kurzerhand zu Stein verwandelt und umgeben seither die Stadt Oruro als felsige Monumente.

Im 16. Jahrhundert kamen die Spanier und mit ihnen der Katholizismus, der sich mit den alten Mythen als kompatibel erwies. Nusta wurde zur Virgen de Socavón, zur Jungfrau der Minen, während Huari fortan als Teufel auftrat.
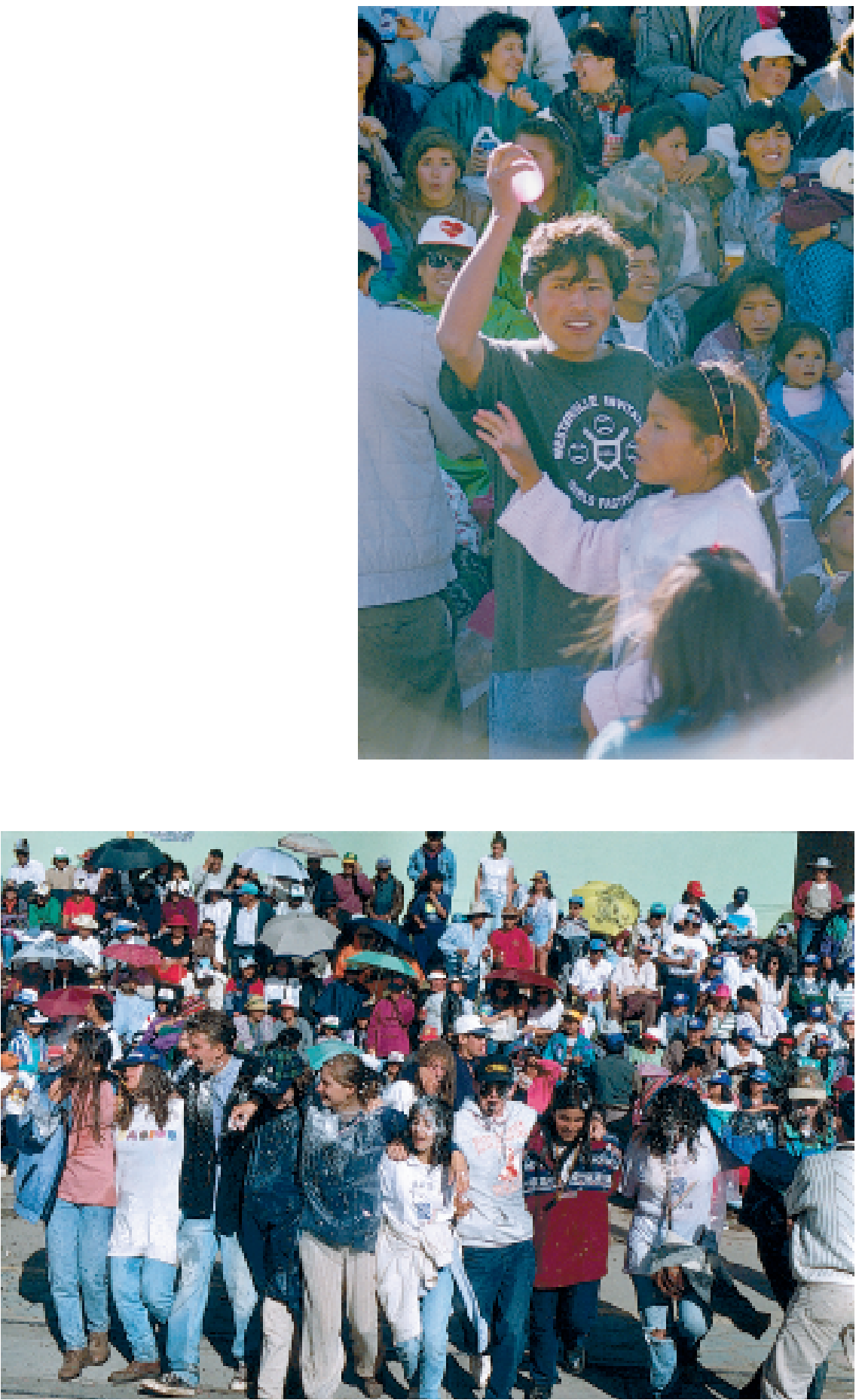

Spass garantiert: Der wassergefüllte Ballon wird sein Ziel finden (oben) - rasierschaumbespritzte Jugendliche im Festfieber (unten).
Dieser Mythos bildet den Hauptstrang eines ganzen Geflechts von Motiven, die im Karneval von Oruro in einer einwöchigen Eruption der Farben, Formen und Töne zum Leben erwachen.

\section{Schauplatz überschäumender Lebensfreude}

Zur Karnevalszeit in der zweiten Februarhälfte findet in Oruro regelmässig eine wundersame Verwandlung statt. Das verschlafene Nest, dessen Einwohner zu über 90 Prozent indianischen Ursprungs sind, wird zu einem Schauplatz überschäumender Lebensfreude. Das letzte Loch ist ausgebucht, und auf Plätzen, wo sonst stoische Einheimische mit ausdruckslosen Gesichtern in einer Art agonischer Starre verharren, wird getanzt, gesungen und gelacht. Selbst ein mumifiziert wirkender Taxifahrer kann während der Fahrt durch das Getümmel unvermittelt zur Wasserspritzpistole greifen, um einem Bekannten durch das offene Fenster hindurch en passant eine Abkühlung zu verpassen. Vor Rasierschaum und wassergefüllten Ballons ist in diesen Tagen niemand sicher, wobei Gringos und hübsche junge Mädchen besonders begehrte Zielobjekte darstellen. Kurzum, man beginnt zu verstehen, warum selbst Cambas - so nennen sich die lebenslustigen und nicht eben altiplanofreundlichen Tieflandbewohner Boliviens - mit Blick auf Oruro vom besten Karneval der Welt schwärmen.

Im Zentrum der Festivitäten steht die Diablada, die Teufelsparade. Ein Name, der Sinn macht, obschon am Schluss des Zugs durch die Stadt das Gute in Form der Jungfrau von Socavón und des Erzengels Michael, der sich irgendwie in die Überlieferung hineingemischelt hat, über das Böse triumphiert. Doch die Show gehört den in Legionsstärke und farbenprächtigen Kostümen wilde Tänze vollführenden Teufeln, die gleich von drei prominenten Vertretern angeführt werden: Luzifer, Satan und der Lokalgrösse China Supay. Am Endpunkt der Parade, auf der Plaza Socavón, macht Erzengel Michael in einem allegorischen Akt den sieben Todsünden - Stolz, Habgier, Wollust, Zorn, Völlerei, Neid und Faulheit - den Garaus.

Die Teufel markieren nur den Auftakt einer fast endlosen Serie von Umzügen, in denen im Verlauf von rund einer Woche zentrale Themen aus der Geschichte und Mythologie der Region in Form ausgelassener Tänze aufgerollt werden. «Los Morenos» greift das Schicksal der von den Spaniern eingeschleppten Negersklaven auf, deren Nachkommen heutzutage vorwiegend in den Yungas - einer subtropischen Region im Departement La Paz - leben. Ihre brutalen Auf- 
seher, «Los Caporales», kommen in der nach ihnen benannten Parade zum Zug. Die «Llamadera» erinnert in tänzerischer Form an die Lamakarawanen, mit denen Urvölker wie die Urus oder Charcas den Inkas Geschenke überbrachten. «Wacatokiris» verulkt die spanische Tradition des Stierkampfs, und «La Challa» ist ein autochthones Ritual, in dessen Rahmen Pachamama Mutter Erde - symbolische Gaben überreicht werden. Jeder Tanz hat seine spezifische musikalische Form, traditionelle Klänge und Rhythmen von eigenwilliger Schönheit, $\mathrm{zu}$ deren bekanntesten Vertretern Huayño, Kullawuada, Saya, Caporal, Cueca, Morenada und Chunsunqui zählen.

\section{Ströme von Bier und Härterem}

Schlaf? Ein Programmpunkt, der zur Karnevalszeit auf der Prioritätenliste ganz unten zu finden ist. Im Freien ist es zu kalt dazu, in den Hotels zu lärmig. Die Ströme von Bier und Härterem, die in diesen Tagen unvermeidlicherweise fliessen und auch ihre tragischen Auswirkungen haben, sind immerhin dazu angetan, eine Art wohliger Trance zu erzeugen, die einen die Unwirtlichkeit orureñischer Nächte besser ertragen lässt. Ein heisser Tip, um bleierne Glieder wieder auf Vordermann zu bringen, ist ein Besuch der Thermalquellen von Obrajes oder Pazña ausserhalb der Stadt, wo man in einfachen Felsbädern wundervoll vor sich hindösen kann, bevor man sich wieder ins Getümmel stürzt.

Nach einer Woche ist der Spuk vorbei, wie ein Vulkanausbruch, der sich abrupt erschöpft hat. Und als sich auf der Rückfahrt feine Schleier von Nieselregen über die ausgetrocknete und menschenleer daliegende Ebene schieben, steigt gleichzeitig mit den Bildern von vibrierender Vitalität, die mich in den letzten Tagen umgeben haben, im Bewusstsein ein Satz von Goethe auf, der im gleichnamigen Aufsatz über die Natur gesagt hat: «Leben ist ihre schönste Erfindung, und der Tod ist ihr Kunstgriff, viel Leben zu haben.» 